\title{
Optimal Power Control for Energy Harvesting Transmitters in an Interference Channel
}

\author{
Kaya Tutuncuoglu Aylin Yener \\ Wireless Communications and Networking Laboratory (WCAN) \\ Electrical Engineering Department \\ The Pennsylvania State University, University Park, PA 16802 \\ kaya@psu.edu_yener@ee.psu.edu
}

\begin{abstract}
In this paper, optimal power control policies for an interference channel with two energy harvesting transmitters and two corresponding receivers are considered. Energy harvesting transmitters have strict power constraints due to the harvesting process as well as battery capacity constraints. The derived optimal power policies maximize the sum-throughput of a deadline constrained system under such energy and data arrival constraints by utilizing the limited available energy and managing the interference in the channel simultaneously. It is shown that an alternating maximization approach that individually optimizes the power policies for each transmitter in a cyclic manner converges to the optimal policy. The single-user subproblems with data constraints are solved using a generalized directional waterfilling algorithm. A practical distributed algorithm requiring only local energy harvesting information is presented, and its nearoptimal performance is demonstrated through simulations.
\end{abstract}

\section{INTRODUCTION}

Wireless networking with energy harvesting nodes provides mobility, reliability and ease of maintenance as well as being an environmentally friendly alternative. These benefits motivate ubiquitous use of energy harvesting wireless communication devices in the near future.

The design principles of energy harvesting wireless networks are fundamentally different than their traditional counterparts due to the instantaneously available energy constraints. Energy availability is stochastic and uneven throughout operation, and the battery to store the harvested energy is limited in practice. A particularly important network structure is one that addresses the case where multiple energy harvesting transmitters share the wireless medium to communicate to multiple destinations, i.e., a wireless ad hoc network with interference. In this paper, we consider the simplest such setting, the two-user interference channel, and solve the optimum power scheduling problem that maximizes short-term sum throughput of this system under a deadline when the two transmitters obtain the transmission energy by harvesting from ambient sources.

Optimal power policies for energy harvesting nodes have attracted recent interest in the research community. The concept of energy neutrality [1] was utilized in queueing-theoretic work [2], [3] to stabilize energy queues of energy harvesting nodes. In such work, the stored energy is often allowed to grow indefinitely, rendering it inapplicable for nodes with limited battery capacity. An alternative approach enforcing strict energy constraints is considered in [4], where transmission time for a given amount of data is optimized over power allocations that strictly obey a known energy arrival scheme. This work has been subsequently extended to the problem of maximizing transmitted data by a deadline with the addition of a battery capacity constraint in [5], and battery imperfections in [6]. A model incorporating channel fading to these problems is introduced in [7] and solved with a directional water-filling algorithm. More recently, multi-user settings such as the broadcast channel [8], [9], or multiple access channel [10] have also been considered. In contrast, we, in this paper, focus on the scenario with multiple transmitters and multiple receivers, i.e., the interference channel.

The interference channel is a fundamental building block for wireless networks. Consequently, identifying the transmission policies under the energy harvesting scenario for this channel, will furnish us with insights needed for energy harvesting wireless ad hoc network design. A critical issue is the lack of conclusive results on the capacity of the interference channel. For the Gaussian two-user interference channel, the strong interference case was introduced and characterized in [11]. However other cases were only recently and partially solved in [12] restricted to sum-capacity, pointing out that the sumcapacity is notably influenced by the interaction of the transmitters. This makes optimal power allocation in the energy constrained setting of our problem even more interesting and relevant.

The focus of this paper is on short-term throughout optimization in a two-user interference channel with energy harvesting transmitters and data arrivals. The problem of transmitting the maximum total number of bits for a given deadline is considered. First, it is shown that an alternating maximization algorithm converges to the optimal solution for jointly concave rate expressions. The alternating subproblems are then solved using the generalized water-filling algorithm modified similar to the directional water filling in [7] to address energy and additional data constraints. Finally, a distributed algorithm is proposed and its performance is compared to that of the optimal policy. 


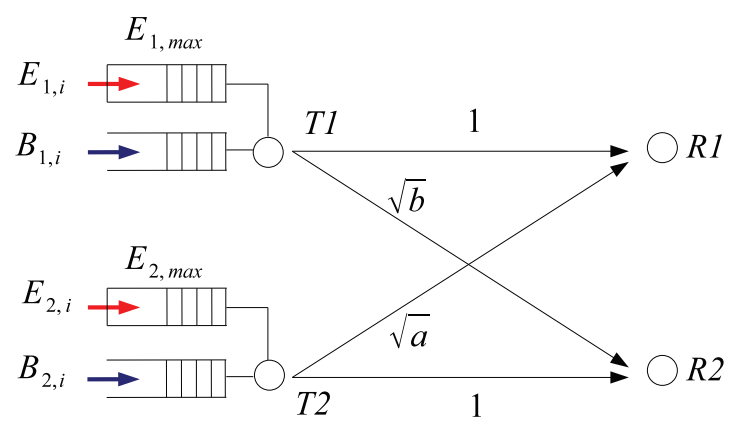

Fig. 1: Interference channel with energy harvesting and data arrivals.

\section{SYSTEM MODEL}

The two-user Gaussian interference channel with energy harvesting transmitters is shown in Figure 1. Transmitters $T 1$ and $T 2$ have independent data packets addressed to corresponding receivers $R 1$ and $R 2$. The transmitters are powered by independent energy harvesting processes, energy from which are stored in batteries of size $E_{1, \max }$ and $E_{2, \max }$ respectively. The harvested energies and battery capacities are normalized to the corresponding transmitter-receiver link gain and receiver noise level, yielding unitary direct channel coefficients and noise variances. After this normalization for each transmitter, the cross channel coefficients become $\sqrt{a}$ and $\sqrt{b}$ and the channel outputs are expressed as

$$
Y_{1}=X_{1}+\sqrt{a} X_{2}+Z_{1}, \quad Y_{2}=\sqrt{b} X_{1}+X_{2}+Z_{1}
$$

where $Y_{1}$ and $Y_{2}$ are received at $R 1$ and $R 2, X_{1}$ and $X_{2}$ are channel inputs by $T 1$ and $T 2$ normalized to have unit channel gains at their corresponding receivers, and $Z_{1}$ and $Z_{2}$ are zero-mean random variables with unit variance. The sumrate achieved by the transmitters consuming powers $p_{1}$ and $p_{2}$ will be referred to as the rate function $r\left(p_{1}, p_{2}\right)$ in the sequel, and corresponding achieved individual rates will be denoted with $r_{j}\left(p_{1}, p_{2}\right)$. For the sake of simplicity, it is assumed that time or state dependent factors which cannot be included in the rate function, such as battery leakage, are ignored.

The energy harvesting process and packet arrival process for node $j \in 1,2$ are denoted in Figure 2 with red and blue arrows respectively. We assume a time slotted system with slots of length $\tau$, where a normalized energy of $E_{j, i}$ units and a data packet of size $B_{j, i}$ bits are received by transmitter $j$ at the beginning of time slot $i$ and is available for immediate use within that time slot. Since an instantaneous energy consumption requires infinite instantaneous power which is impractical, the energy harvests must be stored in the battery before consumption. Thus any arrival exceeding the respective battery capacity is irreversibly lost, and such arrivals are truncated in the model to eliminate trivial battery overflows. Arriving data packets are stored in the data buffer as well, only without a buffer size restriction. For optimal policy analysis, it is assumed that the arrival scheme is perfectly and noncausally known by both transmitters before transmission. This

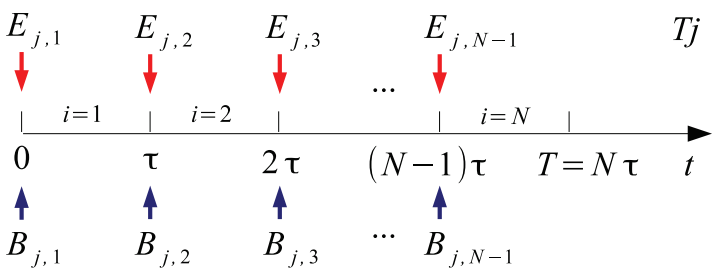

Fig. 2: Energy harvests and data arrivals in the time-slotted model.

problem is referred to as the offline problem. Since such centralized information is not always available, a distributed approach with only local arrival information is put forth in Section V.

There are multiple constraints in this model for a feasible selection of a transmission policy. The first constraint is the energy causality in the sense that no more than the already harvested amount of energy shall be consumed up to a time in transmission. Denoting the transmission power of user $j$ over time slot $i$ as $p_{j, i}$, the constraint for time slot $n$ can be expressed as

$$
\sum_{i=1}^{n} E_{j, i}-\sum_{i=1}^{n} \tau \cdot p_{j, i} \geq 0
$$

where $j$ is the transmitter index chosen from the set $\{1,2\}$. Secondly, it is shown in [5] that a policy with battery overflow is suboptimal since any overflowing energy can be consumed prior to the overflow, strictly increasing the utility as long as data is available. Therefore the battery capacity constraint,

$$
\sum_{i=1}^{n} \tau \cdot p_{j, i}+E_{j, \max }-\sum_{i=1}^{n+1} E_{j, i} \geq 0
$$

is to be met for every $n$ over the transmission. Note that it is possible for a transmitter to not have any extra bits in the data queue when a battery overflow is imminent, rendering overflow avoidance useless. This special case is discussed in detail in Section IV. The final constraint is data causality, implying that no more than the available amount of data can be transmitted until the end of the $n^{\text {th }}$ slot,

$$
\sum_{i=1}^{n} B_{j, i}-\sum_{i=1}^{n} \tau \cdot r_{j}\left(p_{j, i}\right) \geq 0 .
$$

We define the problem of maximizing the total number of bits sent by the transmitter until a deadline $T=N \cdot \tau$, i.e., $N$ time slots, as the short-term throughput maximization problem, which can be expressed as follows:

$$
\begin{array}{cl}
\max _{\boldsymbol{p}_{\mathbf{1}} \geq 0, \boldsymbol{p}_{\mathbf{2}} \geq 0} & \sum_{i=1}^{N} \tau \cdot r\left(p_{1, i}, p_{2, i}\right) \\
\text { s.t. } & (2),(3),(4), \quad n=1, \ldots, N, j \in\{1,2\} .
\end{array}
$$

In (5), the vector $\boldsymbol{p}_{\boldsymbol{j}}$ represents the collection of transmission powers of user $j$, and will be referred to as the power policy or the power allocation vector of user $j$ in the sequel. The 
expression $\boldsymbol{p}_{\boldsymbol{j}} \geq 0$ implies component-wise non-negativity on the transmission power vector.

\section{Convergence of Alternating Maximization}

In this section, we employ an iterative approach to solve the two user optimization problem defined in (5). In particular, we show the convergence of the alternating maximization method where the optimization parameter alternates between the power vectors of the transmitters, namely $\boldsymbol{p}_{\mathbf{1}}$ and $\boldsymbol{p}_{\mathbf{2}}$. Starting from $T 1$ and an arbitrary initial feasible pair $\left(\boldsymbol{p}_{\mathbf{1}}^{\mathbf{0}}, \boldsymbol{p}_{\mathbf{2}}^{\mathbf{0}}\right)$, the following update for the power policies is performed on the $k^{\text {th }}$ iteration:

$$
\begin{aligned}
& \boldsymbol{p}_{\mathbf{1}}^{\boldsymbol{k}}=\underset{\boldsymbol{p}_{\mathbf{1}} \geq 0}{\arg \max } \sum_{i=1}^{N} \tau \cdot r\left(p_{1, i}, p_{2, i}^{k-1}\right) \\
& \text { s.t. } \quad 0 \leq \sum_{i=1}^{n}\left(E_{1, i}-\tau \cdot p_{1, i}\right) \leq E_{1, \max }-E_{1, n+1} \\
& \sum_{i=1}^{n} B_{j, i}-\sum_{i=1}^{n} \tau \cdot r_{j}\left(p_{j, i}\right) \geq 0, j=1,2 \\
& \boldsymbol{p}_{\mathbf{2}}^{\boldsymbol{k}}=\underset{\boldsymbol{p}_{\mathbf{2}} \geq 0}{\arg \max } \sum_{i=1}^{N} \tau \cdot r\left(p_{1, i}^{k}, p_{2, i}\right) \\
& \text { s.t. } \quad 0 \leq \sum_{i=1}^{n}\left(E_{2, i}-\tau \cdot p_{2, i}\right) \leq E_{2, \max }-E_{2, n+1}, \\
& \sum_{i=1}^{n} B_{j, i}-\sum_{i=1}^{n} \tau \cdot r_{j}\left(p_{j, i}\right) \geq 0, \quad j=1,2 \\
&
\end{aligned}
$$

Note that the energy constraints for only the optimized vector is included in each problem. This is possible since the constraints are not coupled due to the fact that the transmitters harvest energy independently. However, this argument is not applicable to the data constraints, since $r_{j}\left(p_{1}, p_{2}\right)$ depends on both power vectors. The problems in (6) and (7) involve single-user optimizations, a similar of which was solved in [13] utilizing a generalized water-filling algorithm. In order to conform to energy and data constraints, this algorithm needs to be enhanced as in [7] with restricted flow dynamics, as will be described in Section IV.

Optimality of the iterative solution is due to the convexity of the problem. The convergence of a coordinate descent method was shown in [14] for a problem of the form

$$
\min f(\boldsymbol{x}) \quad \text { s.t. } \boldsymbol{x} \in \mathcal{X}
$$

where $\mathcal{X}$ is a possibly unbounded box and $f(x)$ is a proper closed convex function. To show this property, we start by stating that for any encoding and decoding scheme achieving a sum-rate $r\left(p_{1}, p_{2}\right)$, there exists a concave achievable sum-rate function $r^{\prime}\left(p_{1}, p_{2}\right)$ that performs at least as good, constructed by utilizing time-sharing of the same algorithm [15].

Lemma 1: Given any transmission scheme achieving an arbitrary rate function $r\left(p_{1}, p_{2}\right)$, one can achieve a rate function $r^{*}\left(p_{1}, p_{2}\right)$ jointly concave in $r_{1}$ and $r_{2}$ that performs at least as good, provided that time-sharing between two power vectors is possible.
Proof: The proof follows from constructing $r^{*}\left(p_{1}, p_{2}\right)$ as $r^{*}\left(p_{1}, p_{2}\right)=\max \left\{\begin{array}{c}\lambda r\left(p_{1}^{\prime}, p_{2}^{\prime}\right)+(1-\lambda) r\left(p_{1}^{\prime \prime}, p_{2}^{\prime \prime}\right) \text { s.t. } \\ \lambda p_{j}^{\prime}+(1-\lambda) p_{j}^{\prime \prime}=p_{j}, j=1,2,0 \leq \lambda \leq 1\end{array}\right\}$ which is by definition concave, and performs as good as $r\left(p_{1}, p_{2}\right)$ since it is included within the maximization set when $\lambda=0$. All rates within the set defined above can be achieved by time-sharing of parameter $\lambda$ between the two power vectors $\left(p_{1}^{\prime}, p_{2}^{\prime}\right)$ and $\left(p_{1}^{\prime \prime}, p_{2}^{\prime \prime}\right)$.

With above lemma, proper closed convexity follows from the null transmission case with $r(0,0)=r_{j}(0,0)=0$ and a bounded transmission power assumption. Note that Lemma 1 can be extended in the same manner to rate functions involving multiple transmitters and receivers.

Theorem 1: The alternating maximization algorithm in $(6,7)$ converges at least linearly.

Proof: By [14, Theorem 2.1], we only need to show the proper closed convexity of the objective function and the Almost Cyclic Rule. The Almost Cyclic Rule requires each coordinate to be iterated once every $B$ iterations. Since the iteration in $(6,7)$ is alternating, this is satisfied for $B=2$.

To show the proper closed convexity of the problem, we first compute the Lagrangian dual function of (5) as in (10), where $\lambda_{j, n}, \mu_{j, n}$ and $\gamma_{j, n}$ are Lagrange multipliers and $C_{\lambda, \mu, \gamma}$ is the collection of the remaining terms that are independent of $\boldsymbol{p}_{\boldsymbol{j}}$. Additional complementary slackness conditions for $\lambda_{j, n}, \mu_{j_{n}}$ and $\gamma_{j, n}$ apply, enabling positive values only when corresponding energy or data constraint is met with equality.

When the additive inverse and thus minimization is considered, the objective function in (11) conforms to proper closed convexity given that $r\left(p_{1}, p_{2}\right)$ and $r_{j}\left(p_{1}, p_{2}\right)$ are concave in $p_{1}$ and $p_{2}$, implying the convergence of the alternating maximization for the Lagrangian problem. The convexity of $-r\left(p_{1}, p_{2}\right)$ is shown in Lemma 1, which reflects to proper closed convexity as discussed previously. The convexity of $-r_{j}\left(p_{1}, p_{2}\right)$ is not trivial for any arbitrary transmission scheme, but is assumed in the sequel since it can be shown to hold for most sum-capacity results in the interference channel. This assumption is only necessary when systems with data arrivals are considered.

When the single coordinate subproblems of the iterative algorithm for the Lagrangian dual is formed, it is observed that these subproblems are identical to the Lagrangian duals of (6) and (7), proving the convergence of the original iterative algorithm proposed.

\section{Solution of Single-User Subproblems}

With the convergence of the single-user iterative algorithm proved, it is necessary to specify how the single user subproblem in each iteration can be solved. For this purpose, we observe the KKT conditions of a single user optimization problem with rate function $r(p)$. The stationarity condition implies that

$\left.\frac{\mathrm{d}}{\mathrm{d} p_{j}}\left(r\left(p_{j}\right)-\sum_{i=n}^{N}\left(\gamma_{j, n} r_{j}\left(p_{j}\right)+\gamma_{\bar{j}, n} r_{\bar{j}}\left(p_{j}\right)\right)\right)\right|_{p_{j, n}}=\sum_{i=n}^{N}\left(\lambda_{j, i}-\mu_{j, i}\right)$. 


$$
\begin{aligned}
\mathcal{L}(\lambda, \mu, \gamma)= & \max _{\boldsymbol{p}_{\mathbf{1}} \geq \mathbf{0}, \boldsymbol{p}_{\mathbf{2}} \geq \mathbf{0}} \sum_{i=1}^{N} \tau \cdot r\left(p_{1, i}, p_{2, i}\right)-\sum_{j=1}^{2} \sum_{n=1}^{N}\left(\lambda_{j, n}\left(\sum_{i=1}^{n}\left(E_{j, i}-\tau \cdot p_{j, i}\right)\right)-\right. \\
& \left.\mu_{j, n}\left(\sum_{i=1}^{n}\left(\tau \cdot p_{j, i}-E_{j, i}\right)+E_{j, \max }-E_{j, n+1}\right)-\gamma_{j, n}\left(\sum_{i=1}^{n}\left(B_{j, i}-\tau \cdot r_{j}\left(p_{j, i}\right)\right)\right)\right) \\
= & \max _{p_{\mathbf{1}} \geq \mathbf{0}, \boldsymbol{p}_{\mathbf{2}} \geq \mathbf{0}} \tau \sum_{i=1}^{N}\left(r\left(p_{1, i}, p_{2, i}\right)-\sum_{j=1}^{2} \gamma_{j, n} \cdot r_{j}\left(p_{j, n}\right)\right)+C_{\lambda, \mu, \gamma}+\tau \sum_{j=1}^{2} \sum_{n=1}^{N} p_{j, n} \underbrace{\left(\sum_{i=n}^{N}\left(\lambda_{j, n}-\mu_{j, n}\right)\right)}_{\beta_{j, n}}
\end{aligned}
$$

needs to be satisfied throughout the transmission, whereas the complementary slackness conditions imply that the Lagrangian multipliers $\lambda_{j, n}, \mu j, n$ and $\gamma_{j, n}$ are positive only when the corresponding constraint is active, and zero otherwise. Moving the data causality terms with multiplier $\gamma_{j, n}$ to the other side of the equation, we observe that the solution can be considered as a generalized water-filling type as in [13], with the multipliers acting to change the desired water level among time slots whenever a constraint is active. The energy causality and battery capacity constraints for this problem have been introduced in [7], yielding a directional water-filling solution where water, corresponding to harvested energy, is allowed to flow only in forward direction and by a limited amount starting from its arrival point. In this paper, we introduce the following flow dynamic to accommodate the new data arrival constraints.

Note that due to the nature of power-rate functions, the derivative of $\gamma_{j, n} r_{j}\left(p_{j}\right)$ in (12) is nonnegative and that of $\gamma_{\bar{j}, n} r_{\bar{j}}\left(p_{j}\right)$ is nonpositive. This indicates that a violated or active data causality constraint for user $j$ at time slot $n$ will act to decrease the power in the prior time slots while that for user $\bar{j} \neq j$ will act in the opposite manner. In the directional water-filling algorithm, this can be interpreted as an additional "pump" element between time slots, forcing water flow in the forward direction when data causality for the user in consideration is violated, or backward when data causality for the other user is. An example is shown in Figure 3, where for small values of $B_{1}$ the pump between the two time slots is activated (b), and remains active until the water level in the first time slot yields a transmission power departing exactly $B_{1}$ bits.

An interesting outcome of the pump modification is the possible contradiction of algorithm elements. Due to the limited battery capacity, it is possible that the pump and tap elements may be active at the same time, the former forcing water flow and the latter resisting it. An example of such cases arise when $E_{1}=E_{2}=E_{\max }$ and $B_{1}=0$ in Figure 3 In such cases, the contradiction is resolved by removing the pumped water until the data constraint is met, in a way allowing the battery to overflow. This does not affect the optimality of the policy since in such a contradicting scenario, the discarded energy is bound to be lost.

\section{Distributed Algorithm}

The optimal policies calculated using the proposed iterative algorithms require the knowledge of energy and data arrival

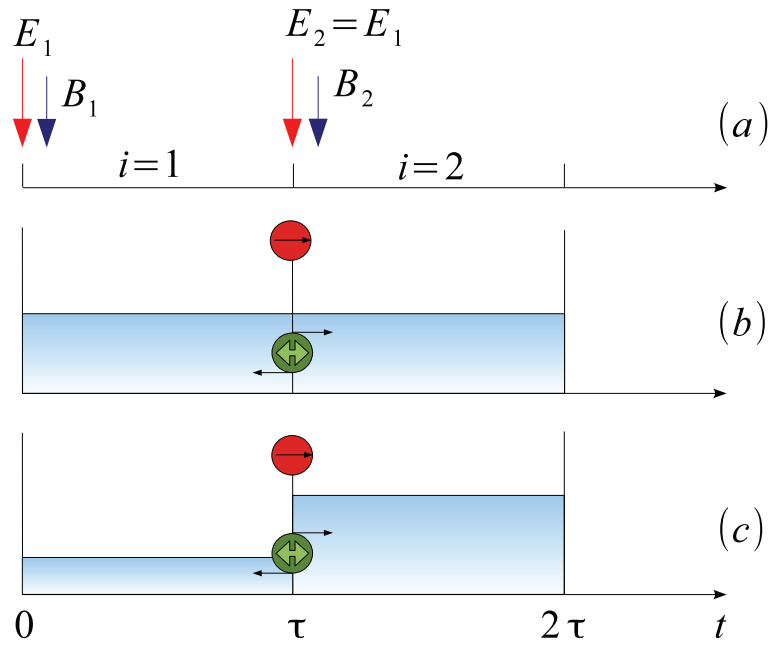

Fig. 3: The operation of the pump element. (a) Arrival scenario, (b) initial water placement without constraints, and (c) optimal allocation after pump is activated.

settings of both transmitters at a centralized controller prior to the transmission to perform the iterations. In practice, such information may not be available or may not be desired to be shared. In this section we propose a near-optimal algorithm that works in a distributed fashion using the insight gained from the centralized optimal iterative solution.

An important result of this paper is the convergence of single user iterative algorithm, and for some sum-capacity achieving schemes it can be seen that the single-user subproblems can further simplify or be independent of the other user. The role of the single user optimization problem in the optimal offline solution indicates that when the model is restricted to localized power decisions at each transmitter, i.e., without any knowledge about the energy or data arrivals of other transmitters, a reasonable algorithm is to determine policies using a single link water-filling approach while assuming expected values for the unknown parameters. For the very strong interference case, this algorithm yields the optimal offline policy; whereas in weaker interference cases, further iterations only provide gradual improvements on the sum rate. Therefore, this simplified approach performs near-optimal, as demonstrated through simulations in Section VI. 


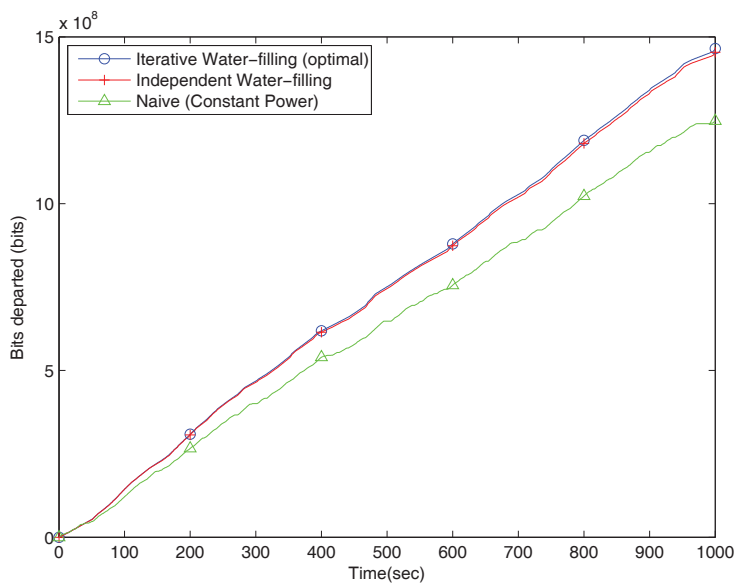

Fig. 4: Simulation of iterative directional water-filling, single user directional water-filling and naive algorithms in asymmetric interference setting with $a=0.7, b=5$ and bandwidth $1 \mathrm{MHz}$.

\section{Simulations}

We now present simulations of the iterative algorithm and distributed algorithm proposed in this paper. We compare the performances of the optimal iterative algorithm, the distributed near-optimal directional water-filling suggested in Section V, and naive nodes that do not perform any kind of algorithm to adapt the energy harvesting process. The naive nodes attempt constant power transmission with the expected energy harvest rate at each time slot if sufficient energy is available, and transmit with all remaining energy otherwise. We assume a Gaussian interference channel with receiver noise spectral density $N_{0,1}=N_{0,2}=10^{-19} \mathrm{~W} / \mathrm{Hz}$, bandwidth $1 \mathrm{MHz}$ and channel coefficients $h_{11}=h_{22}=$ $-100 \mathrm{~dB}, h_{12}=-101.55 \mathrm{~dB}$ and $h_{21}=-93.01 \mathrm{~dB}$ yielding channel parameters $a=0.7$ and $b=5$ after normalization, falling in the asymmetric interference region. For battery capacities $E_{\max , 1}=E_{\max , 2}=10 \mathrm{~mJ}$, we generate energy arrivals with energy distributed uniformly in $\left[0, E_{\max }\right]$ and interarrival times distributed exponentially with mean $5 \mathrm{sec}$, quantized to time slots of duration $\tau=1 \mathrm{sec}$. For this setting, the cumulative departures of these algorithms are plotted in Figure 4. It is apparent that the water-filling algorithms provide notable performance increase over the naive approach. Moreover, it is observed in this simulation as well as others with different parameters that the single user directional waterfilling performs very close to optimal, making it a favorable candidate for practical applications.

\section{CONCLUSION}

In this paper, the short term sum-throughput maximization problem for a two-user interference channel with energy harvesting nodes and data arrivals was formulated and solved with an iterative algorithm. It was observed that the resulting single user subproblems can be solved with generalized directional water-filling algorithm with the addition of a pump element to account for the data causality constraints. With the insight from the optimal solution, a distributed version of the solution was suggested. The performance of the suggested iterative directional water-filling algorithm and its distributed near-optimal counterpart were verified through simulations, showing a notable performance boost over naive algorithms even in the two user case without data arrivals.

Being the building block for multi-user interference networks, the results for the interference channel serve as a starting point for energy harvesting multiple transmitter-receiver networks. This addresses practical interests in analyzing and optimizing the upcoming generation of energy harvesting networks. Future directions for this topic would be extensions to multi-hop network structures as well as online algorithms to adapt to energy availability and interference levels simultaneously.

\section{REFERENCES}

[1] A. Kansal, J. Hsu, S. Zahedi, and M. B. Srivastava, "Power management in energy harvesting sensor networks," ACM Transactions on Embedded Computing Systems (TECS) - Special Section LCTES, vol. 6, no. 4, Sept. 2007.

[2] V. Sharma, U. Mukherji, V. Joseph, and S. Gupta, "Optimal energy management policies for energy harvesting sensor nodes," IEEE Transactions on Wireless Communications, vol. 9, no. 4, pp. 1326-1336, 2010.

[3] M. Gatzianas, L. Georgiadis, and L. Tassiulas, "Control of wireless networks with rechargeable batteries," IEEE Transactions on Wireless Communications, vol. 9, pp. 581-593, Feb. 2010.

[4] J. Yang and S. Ulukus, "Optimal packet scheduling in an energy harvesting communication system," IEEE Transactions on Communications, submitted Jun. 2010, available at http://arxiv.org/abs/1010.1295.

[5] K. Tutuncuoglu and A. Yener, "Optimum transmission policies for battery limited energy harvesting nodes," IEEE Transactions on Wireless Communications, submitted Sept. 2010, available at http://arxiv.org/abs/1010.6280.

[6] B. Devillers and D. Gunduz, "A general framework for the optimization of energy harvesting communication systems with battery imperfections," Arxiv preprint arXiv:1109.5490, 2011.

[7] O. Ozel, K. Tutuncuoglu, J. Yang, S. Ulukus, and A. Yener, "Transmission with energy harvesting nodes in fading wireless channels: Optimal policies," IEEE Journal on Selected Areas in Communications, vol. 29, no. 8, pp. $1732-1743$, Sep. 2011

[8] J. Yang, O. Ozel, and S. Ulukus, "Broadcasting with an energy harvesting rechargeable transmitter," IEEE Transactions on Wireless Communications, submitted Oct. 2010, available at http://arxiv.org/abs/1010.2993.

[9] M. Antepli, E. Uysal-Biyikoglu, and H. Erkal, "Optimal packet scheduling on an energy harvesting broadcast link," IEEE Journal on Selected Areas in Communications, vol. 29, no. 8, pp. 1721 -1731, Sept. 2011.

[10] J. Yang and S. Ulukus, "Optimal packet scheduling in a multiple access channel with rechargeable nodes," in Proceedings of the IEEE International Conference on Communications, ICC'11, Kyoto, Japan, Jun. 2011

[11] H. Sato, "The capacity of the gaussian interference channel under strong interference (corresp.)," IEEE Transactions on Information Theory, vol. 27, no. 6, pp. 786-788, 1981.

[12] X. Shang, G. Kramer, and B. Chen, "A new outer bound and the noisyinterference sum-rate capacity for gaussian interference channels," IEEE Transactions on Information Theory, vol. 55, no. 2, pp. 689-699, 2009.

[13] O. Kaya and S. Ulukus, "Achieving the capacity region boundary of fading CDMA channels via generalized iterative waterfilling," IEEE Transactions on Wireless Communications, vol. 5, no. 11, pp. 32153223, 2006.

[14] Z. Q. Luo and P. Tseng, "On the convergence of the coordinate descent method for convex differentiable minimization," Journal of Optimization Theory and Applications, vol. 72, no. 1, pp. 7-35, 1992.

[15] K. Tutuncuoglu and A. Yener, "Sum-rate optimal power policies for energy harvesting transmitters in an interference channel," JCN Special Issue on Energy Harvesting in Wireless Networks. 